\title{
The Impact of Adherence to the Traditional Mediterranean Diet and Sex Differences on Global Cognitive Functioning: a Systematic Review and Meta-analysis
}

\author{
Michelle E. Kelly ${ }^{1}$ (D) David G. Loughrey ${ }^{2} \cdot$ Joanna McHugh Power $^{1} \cdot$ Claire McEvoy $^{3} \cdot$ Corina Sheerin $^{1} \cdot$ Brian Pennie $^{2}$
}

Received: 3 May 2019 / Accepted: 5 July 2019/Published online: 22 July 2019

(C) Springer Nature Switzerland AG 2019

\begin{abstract}
Contradictory findings in reviews that assess the relationship between the Mediterranean Diet (MedDiet) and cognitive functioning have been attributed to heterogeneity in the criteria used to assess MedDiet and cognition, and differences in the location or cultural habits of the populations studied. Few reviews have examined the relationship between dietary adherence and cognition or considered the impact of sex differences. This systematic review and meta-analysis examines the relationship between adherence to the MedDiet and cognitive functioning of healthy older adults (50+). We included isolated data from Mediterranean regions and considered differential outcomes based on sex. A search of PubMed, Cochrane Library, EMBASE, Scopus and Web of Science from 2011 to 2018 identified five longitudinal cohort studies $(n=3368)$ and one randomized controlled trial (RCT; $n=239$ intervention; $n=95$ controls). The primary outcome of interest was global cognitive functioning. Meta-analysis of cohort studies revealed a significant association between MedDiet adherence and cognitive functioning $(n=$ $3368, r=0.09, p=0.012$ ), an effect greater than previously reported. Meta-regression demonstrated that the effect size was stronger with improved quality of study reporting $(p=0.01)$. Sex did not impact cognitive outcomes, but sex differences in levels of adherence were reported in individual studies. There might be a stronger association between MedDiet and cognitive functioning for older adults from Mediterranean countries compared to other geographical locations, perhaps due to higher adherence and/or longer exposure over the life course. Results are compared to those of prior meta-analyses, and the impact of sources of heterogeneity is considered. The potential influence of sex and gender, as biological and social constructs, on dietary adherence is discussed.
\end{abstract}

Keywords Systematic review $\cdot$ Meta-analysis $\cdot$ Mediterranean Diet $\cdot$ Cognitive functioning $\cdot$ Sex differences

Electronic supplementary material The online version of this article (https://doi.org/10.1007/s41465-019-00143-6) contains supplementary material, which is available to authorized users.

Michelle E. Kelly

Michelle.Kelly@ncirl.ie

David G. Loughrey

loughred@tcd.ie

Joanna McHugh Power

Joanna.Power@ncirl.ie

Claire McEvoy

c.mcevoy@qub.ac.uk

Corina Sheerin

Corina.Sheerin@ncirl.ie
Brian Pennie

pennieb@tcd.ie

1 National College of Ireland, Mayor Street Lower, IFSC, Dublin 1 , Ireland

2 Global Brain Health Institute, Institute of Neuroscience, Trinity College Dublin, Dublin 2, Ireland

3 Centre for Public Health (Institute for Global Food Security), Queen's University Belfast, Grosvenor Road, Belfast, Northern Ireland BT12 6BJ 


\section{Introduction}

The number of people affected by dementia worldwide is expected to triple to 135 million by 2050 (Prince et al. 2013) largely due to increased life expectancy and population ageing. Reducing the prevalence of dementia in older populations has become a critical public health issue (Wu and Sun 2017). With limited pharmacological treatments available (Alzheimer's Association 2015), effective and feasible strategies are urgently required to improve brain health and prevent or delay dementia in older adults. Interventions aimed at improving brain health through modifiable lifestyle factors, such as diet, have become particularly relevant (Lourida et al. 2013). A recent publication by the Lancet Commission identified nine modifiable risk factors to reduce incident dementia, three of which are metabolic factors (Yaffe 2007) (hypertension, obesity, and diabetes) that have clear associations with diet (Livingston et al. 2017).

Adherence to the Mediterranean Diet (MedDiet) reduces vascular and metabolic risk factors (Livingston et al. 2017; Scarmeas et al. 2009), and may be an important dietary strategy for reducing the risk of progression from mild cognitive impairment to dementia (Cooper et al. 2015). The MedDiet refers to the traditional eating habits of those living in Greece, Spain, Italy, and Southern France (De Lorenzo et al. 2010; Grosso and Galvano 2016; Willett et al. 1995) and is characterized by high intake of fresh fruit and vegetables, nuts, cereals, seeds, and legumes, as well as moderate alcohol consumption (specifically red wine) and a low intake of meat (Hardman et al. 2016). The MedDiet has been associated with positive brain health outcomes in some older populations (Morris et al. 2015; Tangney et al. 2011, 2014; Koyama et al. 2015; Feart et al. 2009; Tsivgoulis et al. 2013), but findings have not been entirely consistent (Samieri et al. 2013; Olsson et al. 2015; Vercambre et al. 2012). Longitudinal studies (Morris et al. 2015; Tangney et al. 2011, 2014; Katsiardanis et al. 2013; Martinez-Lapiscina et al. 2013) and randomized control trials (RCTs) (VallsPedret et al. 2015; Kesse-Guyot et al. 2013) have reported diminished rates of cognitive decline, a reduced risk in developing Alzheimer's disease (AD), and better cognitive performance compared to controls, in older adults adhering to MedDiet patterns. In contrast, several studies have shown no association between adherence to the MedDiet and cognitive functioning (Cherbuin and Anstey 2012; Knight et al. 2017; Loughrey et al. 2017).

Recent reviews and meta-analyses (Wu and Sun 2017; Hardman et al. 2016; Petersson and Philippou 2016; Masana et al. 2017; Kyrozis et al. 2013; Wardle et al. 2004) have suggested that contradictory findings between studies may be attributable to heterogeneity in the methods and/or criteria used to assess both MedDiet and cognition, as well as differences in the geographical location or cultural habits of the populations studied. Hence, an examination of data examining adherence to MedDiet and cognitive function taken from countries that are homogenous, for instance, in their typical adherence to the traditional MedDiet, would be valuable (Arganini et al. 2012). Only two reviews to date have examined the relationship between adherence to the MedDiet and cognitive outcomes (Wu and Sun 2017; Hardman et al. 2016). In one review, higher MedDiet adherence was associated with a lower risk of developing mild cognitive impairment, $\mathrm{AD}$, and dementia in nine cohort studies (Wu and Sun 2017), while another reported that greater adherence to the MedDiet was associated with better cognitive function in 18 longitudinal and prospective studies (Hardman et al. 2016). Although most reviews extract information on participants' sex, few examine sex differences in MedDiet adherence or cognitive outcomes.

Sex differences in health research are often neglected (Mattioli et al. 2013). Given the multiplicity of factors that may affect diet adherence, it is essential that sex-specific rather than generic investigations are undertaken and that interventions are tailored towards specific groups. Overall, women tend to have better dietary habits than men (Iaccarino Idelson et al. 2017) and women have shown greater adherence to the MedDiet compared to men in relation to heart health (ProustLima et al. 2008) and in child/teenage samples (Mielke et al. 2014). In relation to cognitive outcomes, women are thought to experience faster decline in cognition with age (Chene et al. 2015) and faster conversion from mild cognitive impairment (MCI) to AD (Kukull et al. 2002) and have a greater risk for dementia, compared to men (Hebert et al. 2001; Brayne et al. 1997; Roberts et al. 2012; Anastasiou et al. 2017), although some inconsistent findings have also been reported (Liberati et al. 2009). It is not yet known, however, whether sex differences exist in relation to the MedDiet and cognitive outcomes in older adults.

This review will update the literature by (i) conducting an analyses of isolated data from Mediterranean regions, where adherence to the traditional MedDiet is likely to be higher than in other non-Mediterranean countries (von Elm et al. 2008); (ii) including data from RCTs as well as cohort studies; and (iii) including an analysis of differential outcomes based on sex. In included studies, global cognitive functioning was measured using composite measures of cognitive function. We focused only on global cognitive functioning as outcome measures in studies are more likely to be homogenous, and because a prior review suggested that the strongest evidence exists for a relationship between the MedDiet and global cognitive functioning (Masana et al. 2017). The aim of this review therefore is to assess the influence of adherence to the MedDiet on measures of global cognitive functioning in the Mediterranean regions of Greece, Spain, Italy, and Southern France. 


\section{Methods}

\section{Search Strategy}

The following databases were searched to identify studies written in English and published from January 2011 up to January 2018: PubMed, the Cochrane Library, EMBASE, Scopus, and Web of Science. Database searches were supplemented with included studies from similar systematic reviews published since 2011. The search strategy (Supplemental Table 1) involved combining key terms relating to cognition (e.g. "cognitive function", "cognition", "brain health") with specific words for the MedDiet (e.g. "Mediterranean"), and key words for older adults (e.g. "old", "older", elderly", "ageing"). Titles and abstracts were screened by two independent reviewers (BP and MK) and cross-checked by DL to exclude articles that did not meet the inclusion criteria. Full texts of the remaining studies were then screened to identify eligible studies (study selection flowchart, Fig. 1). We did not publish a protocol for this review.

\section{Selection Criteria}

We followed the Preferred Reporting Items for Systematic Reviews and Meta-Analysis (PRISMA) guidelines. Studies were included that investigated the effects of the MedDiet on the cognitive functioning of older adults $(50+)$ without known cognitive impairment. Only longitudinal prospective cohort studies and RCTs were eligible for inclusion because they lend themselves better to causal inference than crosssectional studies. Exclusion criteria included studies that investigated hybrid Mediterranean-style diets and/or isolated components of the MedDiet (see excluded studies table, Supplemental Table 2). Only studies conducted in Mediterranean regions that traditionally adhere to a MedDiet were included (Greece, Spain, Italy, and Southern France). The outcomes of interest were adherence to the MedDiet and scores on global measures of cognitive functioning. The following data were extracted from each study: authors and publication year, study design and duration, Mediterranean country of origin, participant gender and age, sample size, MedDiet definitions and assessments used, global cognitive
Fig. 1 PRISMA flow diagram

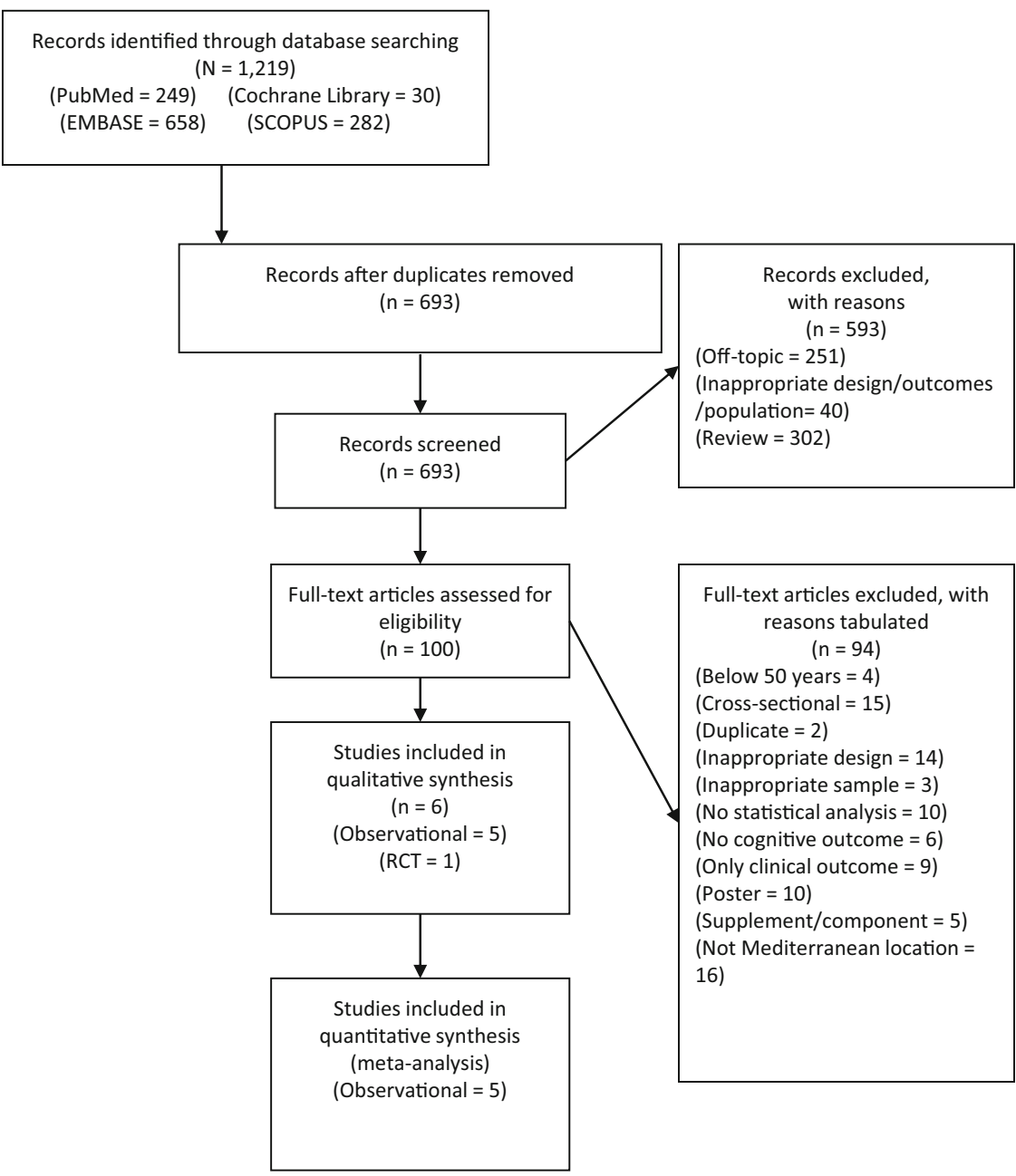


functioning outcome measure, and key results (Tables 1 and 2). Study quality bias was assessed using the Strengthening the Reporting of Observational Studies (STROBE) tool for cohort studies (3 CM-Asv, Biostat 2015) (Supplemental Table 3) and the Cochrane Risk of Bias Assessment for RCTs (Higgins and Green 2011) (Supplemental Table 4).

\section{Statistical Analysis}

Two independent reviewers (DL and MK) extracted the data. Comprehensive Meta-Analysis (CMA) software version 3.0 was used for data analysis (DL). For cohort studies, Pearson's $r$ correlation coefficient was selected to identify studies yielding a positive bivariate association, indicating that MedDiet was associated with improved global cognitive functioning. All effect sizes were first converted to Fisher's $Z$ and then to $r$. For continuous predictor variables, unstandardized $b$ values were standardized by dividing them by the SE, where available. If the SE was not available, the $p$ value was used to estimate the correlation. Standardized $\beta$ values were converted to $r$ by dividing them by the square root of the sample size. For categorical predictor variables, $\beta$ values were entered into CMA either as raw mean differences or as Cohen's $d$ and converted to Fisher's $Z$. ORs were converted to Fisher's $Z$ in CMA and then to $r$. Subgroups in cohort studies (e.g. tertiles of MeDi scores) were combined. Individual effect sizes were combined using the inverse variance random-effects method (DerSimonian and Laird 1986) to facilitate the consideration of heterogeneity among studies. Heterogeneity was examined using $Q$, and any $p$ value $\leq 0 \cdot 10$ was considered statistically significant (Higgins et al. 2003). Inconsistency was examined using I2, and the following grades were applied: $<25 \%$ (very low), 25 to $<50 \%$ (low), 50 to $<75 \%$ (moderate), and $>75 \%$ (large) (Higgins et al. 2003). Priority was given to outcomes that were adjusted for covariates and that removed cognitively impaired participants. Multiple-publication bias was avoided by using data from the most recently published study.

Cumulative meta-analysis ranked by year was used to detect whether there was a dynamic trend in the analysis over time (Lau et al. 1995). Outcomes were analysed with each study deleted from the model once to examine the effects of individual results on the overall findings. Meta-regression was used to examine the potential association between effect size and the following continuous variables: the effects of the length of study, STROBE, age (mean), age (min), and sex (\% female). For categorical variables, we examined between-group differences (between-group $Q$ value) in effect sizes using mixed-effects analysis of variance-like models for meta-analysis if at least 3 effect sizes were available for each category. Small study effects analysis was examined using funnel plots, and the regression-intercept approach of Egger and colleagues (Egger et al. 1997; Sterne et al. 2011) and moderator analysis of the relevant categorical variables were planned where there was sufficient data, i.e. at least 10 for study effects analysis (Egger et al. 1997) and at least 3 effect sizes for moderator analysis were available for each category (Borenstein et al. 2011).

\section{Results}

\section{Included Studies}

The combined search provided a total of 1219 papers. After duplicates were removed and titles and abstracts were reviewed, 1119 papers were excluded, leaving 100 potentially relevant articles. After assessing full texts of the remaining articles, 94 papers were excluded, with reasons recorded. A total of five cohort studies and one RCT involving 4009 participants were included in the review (see Fig. 1 and Tables 1 and 2). Two cohort studies were conducted in Greece (Trichopoulou et al. 2014; Psaltopoulou et al. 2008), one in France (Feart et al. 2009), one in Spain (Galbete et al. 2015), and one in Italy (Gallucci et al. 2013). The RCT was conducted in Spain (Kesse-Guyot et al. 2013). Mean length across all six studies ranged from 2 to 7 years. Studies were published between 2008 and 2015, and all included both sexes with the proportion of women being slightly higher in all but one study (Cherbuin and Anstey 2012; Galbete et al. 2015). Participants across all studies were aged 50 years old and above at baseline, with mean ages ranging from 61.9 to 79.1 . The sample sizes ranged from 309 to 1410 participants.

\section{Assessment of the Mediterranean Diet}

Four of the five prospective cohort studies (Feart et al. 2009; Trichopoulou et al. 2014; Psaltopoulou et al. 2008; Galbete et al. 2015) utilized the initial research by Trichopoulou and colleagues (Trichopoulou et al. 2003, 2014) to calculate MedDiet scores. This original research used sex-specific median cut-off points to assign a value of 0 or 1 to each of nine dietary components of the MedDiet, including vegetables, legumes, fruits and nuts, cereals, fish (beneficial components), meat, poultry, dairy (detrimental components), and ethanol. Depending on consumption and median cut-off points, favourable foods were assigned a value of 0 and unfavourable foods were scored as 1 . Adding the value assigned to each component generated a total MedDiet median score ranging from 0 (minimal adherence) to 9 (maximal adherence). Two of the five cohort studies applied threshold values for adherence of low 0-3, medium 4-5, and high 6-9 (Feart et al. 2009; Trichopoulou et al. 2014), while one study used thresholds of low 0-3, medium 4-6, and high 7-9 (Galbete et al. 2015). One cohort study did not provide information on threshold cut-off scores (Psaltopoulou et al. 2008). The remaining cohort study calculated MedDiet status by asking 


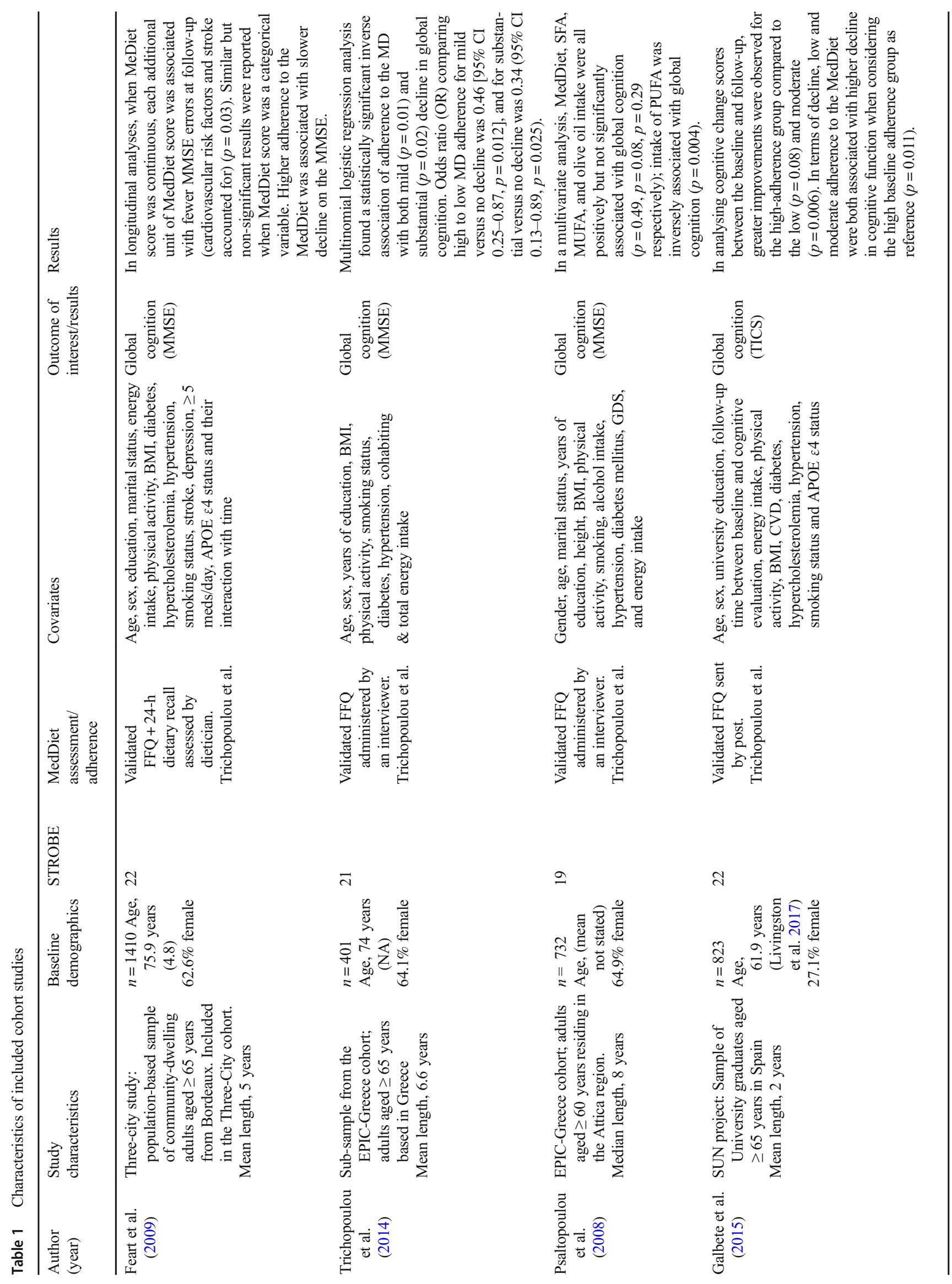




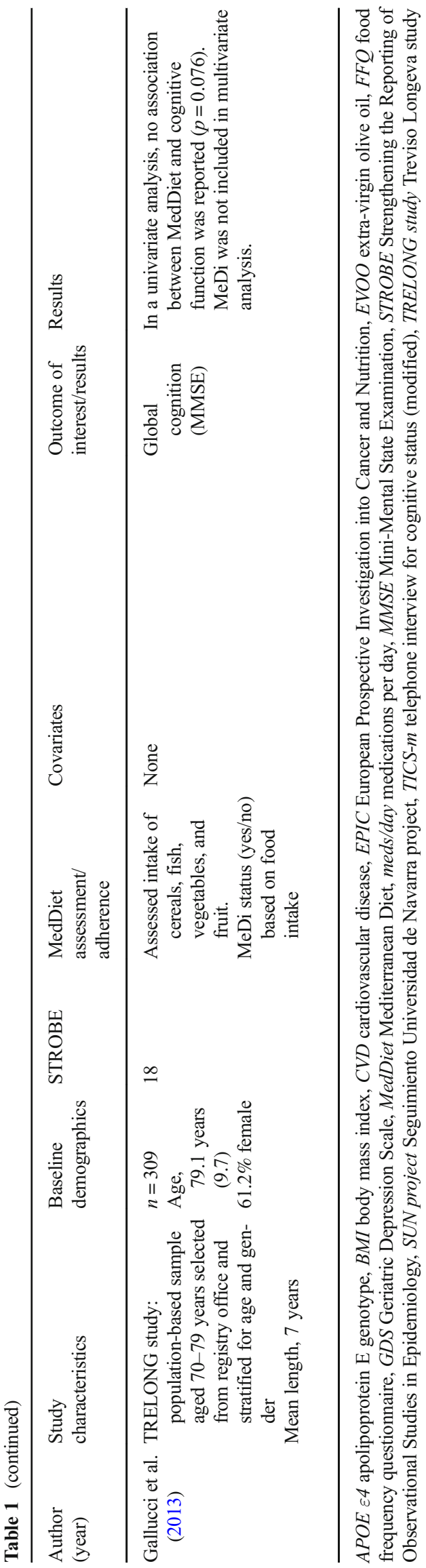

participants whether cereals were usually eaten once a day, vegetables and fruit at least twice a day, and fish at least once a week (yes/no) (Gallucci et al. 2013). In the RCT (KesseGuyot et al. 2013), a validated 14-item screening tool to assess changes and track adherence to the MedDiet was used (Schröder et al. 2011).

\section{Adherence to the Mediterranean Diet}

The mean level of adherence to the MedDiet was low to high across the prospective cohort studies. Adherence was lowest in the Spanish cohort (Galbete et al. 2015), where $33 \%$ of participants were classified with a low score (0-3), 53\% with medium scores (4-6), and $14 \%$ with high scores (7-9). In the French cohort (Feart et al. 2009), 30\% were classified as low (0-3), $44 \%$ as medium (4-5), and $26 \%$ as high (6-9). The greatest level of adherence reported was where $23 \%$ of participants were classified with a low score (0-3), $43 \%$ with medium scores (4-5), and 34\% with high scores (6-9) (Trichopoulou et al. 2014). One cohort study reported an overall rate of $92 \%$ adherence to the MedDiet across their sample, which referred to the fact $92 \%$ of respondents answered yes when asked if they usually ate cereals once per day, vegetables and fruit twice per day, and fish once per week (Gallucci et al. 2013). Information on adherence was not presented in the remaining cohort study (Psaltopoulou et al. 2008).

In the RCT (Kesse-Guyot et al. 2013), participants in the three groups (MedDiet plus olive oil, MedDiet plus nuts, or control) reported similar levels of baseline adherence to the MedDiet. At follow-up, both MedDiet groups had increased adherence to the MedDiet, and pre-post adherence change scores differed significantly between intervention and control groups $(p<0.001)$. Biomarker changes were also assessed, and mean increases of $0.19 \%$ were found for plasma $\alpha$-linolenic acid in the MedDiet plus nuts group and an increase of $49.6 \mu \mathrm{g} / \mathrm{L}$ in urinary hydroxytyrosol in the MedDiet plus olive oil group, which demonstrated high MedDiet adherence.

\section{Assessment of Global Cognitive Functioning}

Four of the five cohort studies (Feart et al. 2009; Trichopoulou et al. 2014; Psaltopoulou et al. 2008; Gallucci et al. 2013) and the RCT (Kesse-Guyot et al. 2013) assessed global cognitive functioning using the Mini-Mental Status Examination (MMSE; Folstein et al. 1975). Domains assessed included orientation to time and place, registration, attention and calculation, recall, language, and visual construction. The remaining study (Galbete et al. 2015) used the Spanish modified version of the telephone interview for cognitive status (TICS-m; 
Gude Ruiz et al. 1994). This version was translated from English to Spanish and has not been formally validated (Galbete et al. 2015). Domains assessed included immediate memory, delayed recall, orientation, attention/calculation, and language.

\section{The Impact of Adherence to the MedDiet on Cognitive Functioning}

In three of the five cohort studies, higher adherence to the MedDiet was significantly associated with reduced cognitive decline (Feart et al. 2009; Trichopoulou et al. 2014; Galbete et al. 2015) and improved cognitive function (Galbete et al. 2015) (see Table 1 for $p$ values). One study (Trichopoulou et al. 2014) examined odds ratios (ORs) and reported that there was a significantly greater risk for mild versus no decline $(\mathrm{OR}=0.46)(p=0.012)$, and for substantial versus no decline $(\mathrm{OR}=0.34)(p=0.025)$ when contrasting high compared to low MD adherence. In the remaining two cohort studies, no association was found between MedDiet and cognitive functioning $(r=0.205)$ (Gallucci et al. 2013), and MedDiet, saturated fatty acid (SFA), monounsaturated fatty acid (MUFA), and olive oil intake were all positively but not significantly associated with global cognitive functioning (unstandardized $\beta$ coefficients of $0.05,0.36$, and 0.26 respectively) (Psaltopoulou et al. 2008). In the RCT (Kesse-Guyot et al. 2013), compared to participants consuming a low-fat control diet, only those consuming a MedDiet supplemented with olive oil had significant improvement in a composite measure of global cognitive functioning after 4 years follow-up.

In examining separate MedDiet components, one cohort study reported that the monounsaturated fatty acid/saturated fatty acid (MUFA/SFA) profile accounted for the significant association between MedDiet and cognitive functioning (Galbete et al. 2015); one reported that MUFA, SFA, and olive oil were positively but not significantly associated with cognitive functioning (Psaltopoulou et al. 2008); and one reported that only vegetable consumption exhibited a significant inverse association with cognitive decline (Trichopoulou et al. 2014). Polyunsaturated fatty acids (PUFA) were also significantly inversely associated with global cognitive functioning (Psaltopoulou et al. 2008). In the RCT, higher consumption of olive oil and nuts was independently related to better cognition, and urinary polyphenol excretion was linearly associated with better memory scores (olive oil and nuts are high in polyphenols) (KesseGuyot et al. 2013).

\section{Sex Differences Across Individual Studies}

Three of the five cohort studies (Trichopoulou et al. 2014; Psaltopoulou et al. 2008; Gallucci et al. 2013) and one 
RCT (Kesse-Guyot et al. 2013) reported no sex differences in MMSE scores, while the remaining two cohort studies (Feart et al. 2009; Galbete et al. 2015) did not consider sex differences in cognitive outcomes. In relation to adherence, two cohort studies reported that men had higher levels of adherence to the MedDiet compared to women (Feart et al. 2009; Psaltopoulou et al. 2008). In one study, women's adherence to MedDiet was also found to be higher than men's, with the exception of nuts, fruit, and dairy (Trichopoulou et al. 2014). No sex differences were evident for nut and fruit consumption, but women consumed more dairy compared to men (Trichopoulou et al. 2014). No significant differences in adherence to MedDiet were apparent in the remaining studies (Kesse-Guyot et al. 2013; Galbete et al. 2015; Gallucci et al. 2013).

\section{Meta-analysis Results}

A meta-analysis of the results from the cohort studies revealed a significant association between the MedDiet and global cognitive functioning ( $p=0.012$, see Fig. 2 and Table 3$)$. There was significant heterogeneity $(Q=16.45$; $p=0.002$ ) and inconsistency was large. In the two analyses with one study removed in each, results were not significant with those of Trichopoulou (Trichopoulou et al. 2014) removed, or with Galbete et al. (2015) removed (Table 4). Cumulative meta-analysis ranked by year found that MedDiet significantly associated with global cognitive functioning since 2015 (Table 4). No small study effects analysis was conducted as there were less than ten effect sizes available for analysis. Meta-regression was used to examine length of study, STROBE, age (mean), age (min), and sex (\% female) (Supplemental Table 5). Only the result for the STROBE assessment was significant $(p=0.01)$, demonstrating that the effect size was stronger with improved quality of study reporting.

\section{Discussion}

This systematic review and meta-analysis evaluated the impact of the traditional MedDiet on measures of global cognitive functioning in 4009 adults aged 50 years and over, without known cognitive impairment and residing in the Mediterranean regions of Greece, Spain, Italy, and Southern France. We identified only one RCT that showed improved cognitive function in response to a MedDiet supplemented with olive oil after 4 years (Kesse-Guyot et al. 2013). The pooled data from five prospective studies demonstrated a positive effect of higher adherence to the MedDiet on measures of global cognitive functioning. The observed pooled effect size was small ( $n=3368, r=0.09$ ), but larger than that reported for MedDiet adherence and global cognition in a recent metaanalysis of cognitively healthy older adults $(n=41,492, r=$ 0.05; Masana et al. 2017). Therefore, it is possible that older adults from Mediterranean countries accrue greater cognitive benefit from the traditional MedDiet, perhaps owing to higher overall adherence and/or longer exposure over the life course. It is important to highlight, however, that MedDiet appears to be beneficial to cognitive functioning irrespective of the country in which it is adopted. Reviews of data from the USA, Australia, France, and Sweden (Wu and Sun 2017) and from the USA, France, Australia, Spain, and Sweden (Hardman et al. 2016) reported an association between MedDiet adherence on cognitive functioning. Despite the limited data available, findings from this review lend support to the adoption of MedDiet for brain health during ageing. These findings are particularly relevant for public health campaigns given the anticipated rise in the number of older people globally that will be affected by cognitive impairment.

Our results in cognitively healthy Mediterranean adults accord with other reviews that have demonstrated an overall beneficial effect of MedDiet on cognitive function in other diverse populations (Wu and Sun 2017; Hardman et al. 2016; Masana et al. 2017; Kyrozis et al. 2013; Wardle et al.

\section{Study name}

Feart et al (2009)
Galbete et al (2015)
Gallucci et al (2013)
Psaltopoulou et al (2008)
Trichopoulou et al (2014)

\begin{tabular}{ccc}
\multicolumn{3}{c}{ Statistics for each study } \\
Correlation & $\begin{array}{c}\text { Lower } \\
\text { limit }\end{array}$ & $\begin{array}{c}\text { Upper } \\
\text { limit }\end{array}$ \\
0.177 & 0.119 & 0.234 \\
0.089 & 0.021 & 0.156 \\
-0.018 & -0.129 & 0.094 \\
0.026 & -0.047 & 0.098 \\
0.156 & 0.061 & 0.248 \\
0.091 & 0.020 & 0.161
\end{tabular}

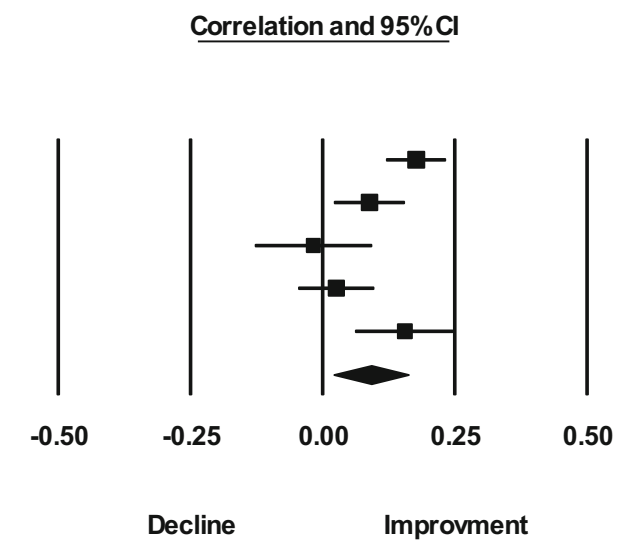

Fig. 2 Forest plots for observational cohort studies showing plots for global cognitive functioning 
Table 3 Mediterranean Diet and global cognitive functioning: main cohort study results

\begin{tabular}{llllllll}
\hline Variable & Studies $(n)$ & Participants $(n)$ & $r$ & $95 \%$ CI & $\mathrm{Z}(p)$ & $\mathrm{Q}(p)$ & $I^{2}(\%)$ \\
\hline $\begin{array}{l}\text { Global cognitive } \\
\text { functioning }\end{array}$ & 5 & 3368 & 0.091 & $0.020,0.161$ & $2.51(0.012)$ & $16.45(0.002)$ & 75.69 \\
\hline
\end{tabular}

2004). Our results remained consistent following metaregression controlling for potential sources of heterogeneity including age, sex, and length of study follow-up. However, the STROBE assessment as a predictor demonstrated that observed effect sizes increased with improved quality of study reporting. This serves as an important reminder of the impact of study reporting quality on meta-analytic results and highlights the need for further high-quality prospective studies in different populations.

We observed no differential effect of sex on the relationship between adherence to MedDiet and global cognitive functioning. Although all studies controlled for sex, sex differences in relation to global cognitive functioning were either not considered (Feart et al. 2009; Galbete et al. 2015) or not significant (Kesse-Guyot et al. 2013; Trichopoulou et al. 2014; Psaltopoulou et al. 2008; Gallucci et al. 2013). Results in relation to sex differences in MedDiet adherence were mixed, with men either found to adhere more (Feart et al. 2009; Psaltopoulou et al. 2008) or less (Trichopoulou et al. 2014) than women, or no differences were reported (Kesse-Guyot et al. 2013; Galbete et al. 2015; Gallucci et al. 2013). Interestingly, gender was not considered in the included studies. This is of relevance as gender and sex, as social and biological constructs, respectively, are distinct and while both are influential on dietary behaviours, gender may be more relevant when considering dietary adherence (Iaccarino Idelson et al. 2017; Butler 2002; Butler and Trouble 1990; Lindeman and Sirelius 2001; Wood and Eagly 2015). Understood as a social construct, gender is malleable, something that we perform and become rather than something we are (Butler 2002; Butler and Trouble 1990). How we enact or "do gender" is influenced by a wide range of factors, including food (Lindeman and Sirelius 2001). For example, meat is often associated with masculinity, and vegetables, dairy, and organic products, with femininity (Shin and Mattila 2019; Sobal 2005). These factors may therefore logically influence whether one adheres to a dietary intervention or not. Despite this, the distinction between gender and sex is not widely considered in the MedDiet literature. To improve our understanding of how gender might influence MedDiet adherence and in turn affect cognitive health outcomes, future research should consider gender and sex as distinct, and examine any differential effects that gender differences may have on relevant outcomes.

Although we attempted to minimize reporting bias in the meta-analysis by including only prospective data from Mediterranean regions, we observed a high degree of heterogeneity between studies, which has also been reported in prior reviews (Wu and Sun 2017; Masana et al. 2017). The followup period of studies included in our review ranged from 2 to 7 years, which is quite broad when considering the trajectory of global cognitive functioning in an ageing population. Given that cognitive function declines slowly and progressively during normal ageing, with high variability in the rate of decline (Blazer et al. 2015), the duration of follow-up in prospective studies is important. The mean age also varied across studies, ranging by almost two decades, which would also have implications when considering cognitive functioning and its decline. Studies with older cohorts, for instance, would be more likely to observe change in cognitive functioning across shorter timeframes since cognitive decline increases in negative slope in the 3 to 6 years before death (Wilson et al. 2003). All six studies included in the meta-analysis used either the MMSE or a measure that was validated against the MMSE, so heterogeneity is unlikely to have arisen in this aspect of study comparison. While included studies allowed a pooled analysis for measures of global cognitive function, examining the effects of MedDiet on specific cognitive domains, from the earliest through to the end stages of decline, could provide greater insight into potential mechanisms of diet on brain health during ageing and help to inform outcome measures for dietary intervention trials targeting brain health.

It was not surprising that the MMSE was the most commonly used measure in the included studies as it has been

Table 4 Mediterranean Diet and global cognitive functioning: results from one study removed and cumulative analysis in cohort studies

\begin{tabular}{llll}
\hline Variable & One study removed & Cumulative analysis & Soint difference smallest/largest (\%) \\
\cline { 2 - 3 } & Study & $0.046(41.1)$ & Significant since \\
\hline $\begin{array}{l}\text { Global cognitive } \\
\text { functioning }\end{array}$ & $\begin{array}{l}\text { Trichopoulou et al. (2014) } \\
\text { Galbete et al. (2015) }\end{array}$ & 2015 & \\
\hline
\end{tabular}


reported to be the most frequently applied cognitive test worldwide (Carnero-Pardo 2014). The widespread use of the MMSE can be controversial due to threats to validity and reliability resulting from misuse of the assessment (Monroe and Carter 2012), its lack of sensitivity to mild deficits and classification inaccuracies (Van Patten et al. 2019), and the considerable effect of socio-educational variables on results (Carnero-Pardo 2014). That said, the advantage of the widespread use of the MMSE in cognitive research is that it allows direct comparisons to be made across studies and contributes to homogeneity in meta-analyses. A Cochrane review concluded that the MMSE could still be used but only as part of a broader assessment battery when results can be interpreted in the context of the individual (Creavin et al. 2016).

The studies in this review used a range of cognitive assessment tools and explored various domains of cognitive functioning, though many were not sufficiently well aligned to allow meta-analysis. This issue has been commented upon previously (Lourida et al. 2013; Hardman et al. 2016). A solution has been offered (Petersson and Philippou 2016), and suggests that future research exploring the link between MedDiet and cognitive functioning should employ a standardized assessment protocol to facilitate future meta-analyses specific to cognitive domains. This is important as different domains may have unique patterns and mechanisms of decline. For example, since semantic memory may decline in early Alzheimer's disease but not in non-pathological age-related cognitive decline (Spaan et al. 2003), it may be of interest to explore the relationship between MedDiet adherence and semantic memory measures (e.g. verbal fluency), since this may be informative specifically about mechanisms through which diet impacts Alzheimer's pathology.

As with all dietary intake studies, measurement error and misclassification bias for dietary exposure are possible. The MedDiet is a priori defined dietary pattern that accounts for complex and synergistic interactions between individual food components; however, different methodologies have been used in individual studies to assess MedDiet adherence. In this meta-analysis, four of the five prospective studies employed a consistent approach to generate MedDiet scores based on population median cut points for individual food items, although only two applied the same threshold cut-off points for medium and high adherence. The remaining study (Gallucci et al. 2013) and the RCT (Kesse-Guyot et al. 2013) used a different approach to derive MedDiet scores. It would be of interest to explore the psychometric properties of the scales used to measure MedDiet adherence particularly since one study used an approach that did not seem to be previously validated (Gallucci et al. 2013). Interestingly, the two studies on which the significance of the overall results depended (Trichopoulou et al. 2014; Galbete et al. 2015) diverged considerably in the rates of adherence to MedDiet reported. This means that it was possible to detect an effect of MedDiet adherence on global cognitive functioning independent of the pattern of adherence detected in the sample. Four of the six studies highlighted specific components of MedDiet that appeared important in predicting global cognitive functioning which included MUFA:SFA (Psaltopoulou et al. 2008; Galbete et al. 2015), olive oil (Kesse-Guyot et al. 2013; Psaltopoulou et al. 2008), and vegetables (Trichopoulou et al. 2014). Interestingly, these food components have been independently shown to be positively associated with brain health outcomes (Morris 2016). Therefore, while the MedDiet has garnered the most attention in the diet-dementia field, the overall dietary pattern may not represent an optimal combination of foods and nutrients for brain health in older adults.

The MedDiet is nutritionally diverse and provides a high MUFA:SFA ratio due to the use of olive oil as the main fat source, fibre, vitamins, folate, and natural antioxidants (Willett et al. 1995; Bach et al. 2006). Mechanistically, the MedDiet is likely to benefit brain health in several ways including the inhibition of oxidative stress and inflammatory pathways implicated in the pathogenesis of cognitive decline and dementia. Adherence to the MedDiet is demonstrated to decrease markers of both oxidative stress (Fito et al. 2007) and systemic inflammation (Casas et al. 2014). Furthermore, experimental data shows direct beneficial effects of MedDiet on the integrity of neuronal cells that contribute to brain health (Dauncey 2009; Bourre 2006). Neuro-imaging biomarkers may provide sensitive measures of cognitive function to predict long-term effects of dietary interventions earlier and help to elucidate potential mechanisms of action (Pistollato et al. 2015). Inverse associations between MedDiet and preclinical brain changes associated with dementia $(\mathrm{Gu}$ et al. 2015; Luciano et al. 2017; Mosconi et al. 2014; Berti et al. 2018) are beginning to emerge, but further prospective data are warranted to identify subtle neurological changes that are amenable to dietary intervention and may not be possible to detect with standard cognitive tests in cognitively healthy populations.

There are methodological limitations in the current analysis that we wish to highlight. We used the inverse variance random-effects method to combine individual effect sizes and to enable us to consider heterogeneity. The use of relative rather than absolute cut-off points may have led to misclassification in the separate studies between various levels of MedDiet adherence, making comparisons across studies difficult to interpret. We also consider methodological strengths in the current analysis. We adhered to PRISMA guidelines in our reporting of selected studies (Higgins and Green 2011). We also chose to focus our efforts on studies that could contribute to causal inference, i.e. RCT and longitudinal prospective studies only (while acknowledging that only well-conducted RCT studies can truly inform us about causality). This means that it is unlikely that the overall effect hereby reported is due 
to reverse causality (the impact of cognitive function on adherence to MedDiet), although of course it does not rule out the possibility that this reciprocal pathway exists. It should also be noted that a greater degree of methodological homogeneity was observed across studies when compared to other reviews on the same topic. However, limitations of methodology remain the most significant limitation in the current review.

\section{Conclusion}

Findings from this review and meta-analysis indicate that higher adherence to the MedDiet may contribute to better global cognitive functioning in older populations living in Mediterranean countries. In comparison to reviews which included non-Mediterranean countries, it appears that greater adherence to the MedDiet was observed in the current review. Future research might consider the use of biomarkers to discern compliance with specific food components among groups, although given threshold effects of nutritional biomarkers, they should be used in conjunction with validated dietary intake assessments in target populations. It is recommended that future research employ standardized measurement protocols to determine both MedDiet adherence and cognitive functioning. Studies should also include a standardized nutritional intake assessment that can be used to establish the impact of cognitive changes over time (Hardman et al. 2016), and examine any differential effects that gender differences may have on the relevant outcomes. Further studies are required to identify an optimal dietary pattern for brain health during ageing. Overall, standardization is required to produce more reliably comparable results across studies and to minimize inconsistencies between various research designs (Kelly et al. 2014).

\begin{abstract}
Acknowledgements All of the authors have read and approved the final manuscript. BP and MEK contributed to the study concept and design. BP, DGL, JMcHP, and MEK contributed to the data collection. DGL and MEK conducted data extraction. DGL conducted the statistical analyses. $\mathrm{BP}, \mathrm{MEK}, \mathrm{JMcHP}, \mathrm{CMcE}$, and CS drafted the manuscript and provided important intellectual content.
\end{abstract}

Funding Sources No funding was provided to write this review. BP is supported by the Irish Research Council Research Masters Scholarship; JMcHP is supported by a Health Research Board (HRB) Applied Partnership Award and a Centre for Ageing Research and Development in Ireland (CARDI) Leadership in Ageing Research Fellowship; and $\mathrm{CMcE}$ is supported by a Beeson-CARDI Fellowship in Aging Research from the American Federation of Aging Research. This review was completed independently of any funded projects.

\section{Compliance with Ethical Standards}

Conflict of Interest The authors declare that they have no conflicts of interest.

\section{References}

3 CM-Asv, Biostat. (2015). BioStat. Comprehensive meta-analysis. NJ, USA: Englewood Cliffs.

Alzheimer's Association. (2015). Alzheimer's association report 2015: Alzheimer's disease facts and figures. Alzheimer's \& Dementia, 11(3), 332-384.

Anastasiou, C. A., Yannakoulia, M., Kosmidis, M. H., Dardiotis, E., Hadjigeorgiou, G. M., Sakka, P., et al. (2017). Mediterranean diet and cognitive health: initial results from the Hellenic longitudinal investigation of ageing and diet. PLoS One, 12(8), e0182048.

Arganini, C., Saba, A., Comitato, R., Virgili, F., \& Turrini, A. (2012). Gender differences in food choice and dietary intake in modern Western societies. In J. Maddock (Ed.), Public Health-Social and Behavioral Health. Hampshire: InTech.

Bach, A., Serra-Majem, L., Carrasco, J. L., Roman, B., Ngo, J., Bertomeu, I., et al. (2006). The use of indexes evaluating the adherence to the Mediterranean diet in epidemiological studies: a review. Public Health Nutrition, 9(1a), 132-146.

Berti, V., Walters, M., Sterling, J., Quinn, C. G., Logue, M., Andrews, R., et al. (2018). Mediterranean diet and 3-year Alzheimer brain biomarker changes in middle-aged adults. Neurology, 90(20), e1789ele98.

Blazer, D. G., Yaffe, K., \& Karlawish, J. (2015). Cognitive aging: a report from the Institute of Medicine. Jama, 313(21), 2121-2122.

Borenstein, M., Hedges, L., Higgins, J., \& Rothstein, H. (2011). Introduction to meta-analysis. London: Wiley.

Bourre, J. M. (2006). Effects of nutrients (in food) on the structure and function of the nervous system: update on dietary requirements for brain. Part 2 : macronutrients. The Journal of Nutrition, Health \& Aging, 10(5), 386-399.

Brayne, C., Best, N., Muir, M., Richards, S. J., \& Gill, C. (1997). Fiveyear incidence and prediction of dementia and cognitive decline in a population sample of women aged 70-79 at baseline. International Journal of Geriatric Psychiatry, 12(11), 1107-1118.

Butler, J. (2002). Gender trouble. New York: Routledge.

Butler, J., \& Trouble, G. (1990). Feminism and the subversion of identity. New York: Routledge.

Carnero-Pardo, C. (2014). Should the mini-mental state examination be retired? Neurologia, 29(8), 473-481.

Casas, R., Sacanella, E., \& Estruch, R. (2014). The immune protective effect of the Mediterranean diet against chronic low-grade inflammatory diseases. Endocrine, Metabolic \& Immune Disorders Drug Targets, 14(4), 245-254.

Chene, G., Beiser, A., Au, R., Preis, S. R., Wolf, P. A., Dufouil, C., et al. (2015). Gender and incidence of dementia in the Framingham Heart Study from mid-adult life. Alzheimer's \& Dementia, 11(3), 310-320.

Cherbuin, N., \& Anstey, K. J. (2012). The Mediterranean diet is not related to cognitive change in a large prospective investigation: the PATH through life study. The American Journal of Geriatric Psychiatry, 20(7), 635-639.

Cooper, C., Sommerlad, A., Lyketsos, C. G., \& Livingston, G. (2015). Modifiable predictors of dementia in mild cognitive impairment: a systematic review and meta-analysis. The American Journal of Psychiatry, 172(4), 323-334.

Creavin, S. T., Wisniewski, S., Noel-Storr, A. H., Trevelyan, C. M., Hampton, T., Rayment, D., et al. (2016). Mini-Mental State Examination (MMSE) for the detection of dementia in clinically unevaluated people aged 65 and over in community and primary care populations. Cochrane Database of Systematic Reviews(1), CD011145. https://doi.org/10.1002/14651858.CD011145.pub2.

Dauncey, M. J. (2009). New insights into nutrition and cognitive neuroscience. The Proceedings of the Nutrition Society, 68(4), 408-415.

De Lorenzo, A., Noce, A., Bigioni, M., Calabrese, V., Della Rocca, D. G., Di Daniele, N., et al. (2010). The effects of Italian Mediterranean 
organic diet (IMOD) on health status. Current Pharmaceutical Design, 16(7), 814-824.

DerSimonian, R., \& Laird, N. (1986). Meta-analysis in clinical trials. Controlled Clinical Trials, 7(3), 177-188.

Egger, M., Davey Smith, G., Schneider, M., \& Minder, C. (1997). Bias in meta-analysis detected by a simple, graphical test. Bmj, 315(7109), 629-634.

von Elm, E., Altman, D. G., Egger, M., Pocock, S. J., Gotzsche, P. C., \& Vandenbroucke, J. P. (2008). The strengthening the reporting of observational studies in epidemiology (STROBE) statement: Guidelines for reporting observational studies. Journal of Clinical Epidemiology, 61(4), 344-349.

Feart, C., Samieri, C., Rondeau, V., Amieva, H., Portet, F., Dartigues, J. F., et al. (2009). Adherence to a Mediterranean diet, cognitive decline, and risk of dementia. Jama, 302(6), 638-648.

Fito, M., Guxens, M., Corella, D., Saez, G., Estruch, R., de la Torre, R., et al. (2007). Effect of a traditional Mediterranean diet on lipoprotein oxidation: a randomized controlled trial. Archives of Internal Medicine, 167(11), 1195-1203.

Folstein, M. F., Folstein, S. E., \& McHugh, P. R. (1975). "Mini-mental state". A practical method for grading the cognitive state of patients for the clinician. Journal of Psychiatric Research, 12(3), 189-198.

Galbete, C., Toledo, E., Toledo, J. B., Bes-Rastrollo, M., Buil-Cosiales, P., Marti, A., et al. (2015). Mediterranean diet and cognitive function: the sun project. The Journal of Nutrition, Health \& Aging, 19(3), 305-312.

Gallucci, M., Mazzuco, S., Ongaro, F., Di Giorgi, E., Mecocci, P., Cesari, M., et al. (2013). Body mass index, lifestyles, physical performance and cognitive decline: the "Treviso Longeva (TRELONG)" study. The Journal of Nutrition, Health \& Aging, 17(4), 378-384.

Grosso, G., \& Galvano, F. (2016). Mediterranean diet adherence in children and adolescents in southern European countries. NFS Journal, 3, 13-19.

Gu, Y., Brickman, A. M., Stern, Y., Habeck, C. G., Razlighi, Q. R., Luchsinger, J. A., et al. (2015). Mediterranean diet and brain structure in a multiethnic elderly cohort. Neurology, 85(20), 1744-1751.

Gude Ruiz, R., Calvo Mauri, J. F., \& Carrasco Lopez, F. J. (1994). The Spanish version and pilot study of a telephone test of cognitive status for evaluation and screening in dementia assessment and follow-up. Atencion Primaria, 13(2), 61-66.

Hardman, R. J., Kennedy, G., Macpherson, H., Scholey, A. B., \& Pipingas, A. (2016). Adherence to a Mediterranean-style diet and effects on cognition in adults: a qualitative evaluation and systematic review of longitudinal and prospective trials. Frontiers in Nutrition, 3,22 .

Hebert, L. E., Scherr, P. A., McCann, J. J., Beckett, L. A., \& Evans, D. A. (2001). Is the risk of developing Alzheimer's disease greater for women than for men? American Journal of Epidemiology, 153(2), $132-136$.

Higgins, J. P., Thompson, S. G., Deeks, J. J., \& Altman, D. G. (2003). Measuring inconsistency in meta-analyses. Bmj, 327(7414), 557560.

Iaccarino Idelson, P., Scalfi, L., \& Valerio, G. (2017). Adherence to the Mediterranean Diet in children and adolescents: a systematic review. Nutrition, Metabolism, and Cardiovascular Diseases, 27(4), 283299.

Higgins, J. P. T., Green, S. (2011). Cochrane Handbook for Systematic Reviews of Interventions Version 5.1.0 [updated March 2011]. The Cochrane Collaboration.

Katsiardanis, K., Diamantaras, A. A., Dessypris, N., Michelakos, T., Anastasiou, A., Katsiardani, K. P., et al. (2013). Cognitive impairment and dietary habits among elders: The Velestino study. Journal of Medicinal Food, 16(4), 343-350.

Kelly, M. E., Loughrey, D., Lawlor, B. A., Robertson, I. H., Walsh, C., \& Brennan, S. (2014). The impact of exercise on the cognitive functioning of healthy older adults: a systematic review and metaanalysis. Ageing Research Reviews, 16, 12-31.

Kesse-Guyot, E., Andreeva, V. A., Lassale, C., Ferry, M., Jeandel, C., Hercberg, S., et al. (2013). Mediterranean diet and cognitive function: a French study. The American Journal of Clinical Nutrition, 97(2), 369-376.

Knight, A., Bryan, J., \& Murphy, K. (2017). The Mediterranean diet and age-related cognitive functioning: A systematic review of study findings and neuropsychological assessment methodology. Nutritional Neuroscience, 20(8), 449-468. https://doi.org/10.1080/ 1028415X.2016.

Koyama, A., Houston, D. K., Simonsick, E. M., Lee, J. S., Ayonayon, H. N., Shahar, D. R., et al. (2015). Association between the Mediterranean diet and cognitive decline in a biracial population. The Journals of Gerontology. Series A, Biological Sciences and Medical Sciences, 70(3), 354-359.

Kukull, W. A., Higdon, R., Bowen, J. D., McCormick, W. C., Teri, L., Schellenberg, G. D., et al. (2002). Dementia and Alzheimer disease incidence: a prospective cohort study. Archives of Neurology, 59(11), 1737-1746.

Kyrozis, A., Ghika, A., Stathopoulos, P., Vassilopoulos, D., Trichopoulos, D., \& Trichopoulou, A. (2013). Dietary and lifestyle variables in relation to incidence of Parkinson's disease in Greece. European Journal of Epidemiology, 28(1), 67-77.

Lau, J., Schmid, C. H., \& Chalmers, T. C. (1995). Cumulative metaanalysis of clinical trials builds evidence for exemplary medical care. Journal of Clinical Epidemiology, 48(1), 45-57 discussion 960.

Liberati, A., Altman, D. G., Tetzlaff, J., Mulrow, C., Gøtzsche, P. C., Ioannidis, J. P. A., et al. (2009). The PRISMA statement for reporting systematic reviews and meta-analyses of studies that evaluate health care interventions: explanation and elaboration. PLoS Medicine, 6(7), e1000100.

Lindeman, M., \& Sirelius, M. (2001). Food choice ideologies: the modern manifestations of normative and humanist views of the world. Appetite, 37(3), 175-184.

Livingston, G., Sommerlad, A., Orgeta, V., Costafreda, S. G., Huntley, J., Ames, D., et al. (2017). Dementia prevention, intervention, and care. The Lancet, 390(10113), 2673-2734.

Loughrey, D. G., Lavecchia, S., Brennan, S., Lawlor, B. A., \& Kelly, M. E. (2017). The impact of the Mediterranean diet on the cognitive functioning of healthy older adults: a systematic review and metaanalysis. Advances in Nutrition, 8(4), 571-586.

Lourida, I., Soni, M., Thompson-Coon, J., Purandare, N., Lang, I. A., Ukoumunne, O. C., et al. (2013). Mediterranean diet, cognitive function, and dementia: a systematic review. Epidemiology, 24(4), 479-489.

Luciano, M., Corley, J., Cox, S. R., Valdés Hernández, M. C., Craig, L. C. A., Dickie, D. A., et al. (2017). Mediterranean-type diet and brain structural change from 73 to 76 years in a Scottish cohort. Neurology, 88(5), 449-455.

Martinez-Lapiscina, E. H., Clavero, P., Toledo, E., Estruch, R., SalasSalvado, J., San Julian, B., et al. (2013). Mediterranean diet improves cognition: The PREDIMED-NAVARRA randomised trial. Journal of Neurology, Neurosurgery, and Psychiatry, 84(12), $1318-1325$.

Masana, M. F., Koyanagi, A., Haro, J. M., \& Tyrovolas, S. (2017). N-3 fatty acids, Mediterranean diet and cognitive function in normal aging: a systematic review. Experimental Gerontology, 91, 39-50.

Mattioli, A. V., Pennella, S., Pedrazzi, P., Rosi, C., \& Farinetti, A. (2013). Gender differences in adherence to Mediterranean diet and risk of atrial fibrillation. European Heart Journal, 34(suppl_1), 4270.

Mielke, M. M., Vemuri, P., \& Rocca, W. A. (2014). Clinical epidemiology of Alzheimer's disease: assessing sex and gender differences. Clinical Epidemiology, 6, 37-48. 
Monroe, T., \& Carter, M. (2012). Using the Folstein mini mental state exam (MMSE) to explore methodological issues in cognitive aging research. European Journal of Ageing, 9(3), 265-274.

Morris, M. C. (2016). Nutrition and risk of dementia: overview and methodological issues. Annals of the New York Academy of Sciences, 1367(1), 31-37.

Morris, M. C., Tangney, C. C., Wang, Y., Sacks, F. M., Barnes, L. L., Bennett, D. A., et al. (2015). MIND diet slows cognitive decline with aging. Alzheimer's \& Dementia, 11(9), 1015-1022.

Mosconi, L., Murray, J., Tsui, W. H., Li, Y., Davies, M., Williams, S., et al. (2014). Mediterranean diet and magnetic resonance imagingassessed brain atrophy in cognitively normal individuals at risk for Alzheimer's disease. The Journal of Prevention of Alzheimer's Disease, 1(1), 23-32.

Olsson, E., Karlstrom, B., Kilander, L., Byberg, L., Cederholm, T., \& Sjogren, P. (2015). Dietary patterns and cognitive dysfunction in a 12-year follow-up study of 70 year old men. Journal of Alzheimer's Disease, 43(1), 109-119.

Petersson, S. D., \& Philippou, E. (2016). Mediterranean diet, cognitive function, and dementia: a systematic review of the evidence. Advances in Nutrition, 7(5), 889-904.

Pistollato, F., Cano, S. S., Elio, I., Vergara, M. M., Giampieri, F., \& Battino, M. (2015). The use of neuroimaging to assess associations among diet, nutrients, metabolic syndrome, and Alzheimer's disease. Journal of Alzheimer's Disease, 48(2), 303-318.

Prince, M., Guerchet, M., \& Prina, M. (2013). Alzheimer's disease international. Policy brief for heads of government: the global impact of dementia 2013-2050. London: Alzheimer's Disease International.

Proust-Lima, C., Amieva, H., Letenneur, L., Orgogozo, J. M., JacqminGadda, H., \& Dartigues, J. F. (2008). Gender and education impact on brain aging: a general cognitive factor approach. Psychology and Aging, 23(3), 608-620.

Psaltopoulou, T., Kyrozis, A., Stathopoulos, P., Trichopoulos, D., Vassilopoulos, D., \& Trichopoulou, A. (2008). Diet, physical activity and cognitive impairment among elders: The EPIC-Greece cohort (European Prospective Investigation into Cancer and Nutrition). Public Health Nutrition, 11(10), 1054-1062.

Roberts, R. O., Geda, Y. E., Knopman, D. S., Cha, R. H., Pankratz, V. S., Boeve, B. F., et al. (2012). The incidence of MCI differs by subtype and is higher in men: The Mayo Clinic Study of Aging. Neurology, $78(5), 342-351$.

Samieri, C., Grodstein, F., Rosner, B. A., Kang, J. H., Cook, N. R., Manson, J. E., et al. (2013). Mediterranean diet and cognitive function in older age. Epidemiology, 24(4), 490-499.

Scarmeas, N., Stern, Y., Mayeux, R., Manly, J. J., Schupf, N., \& Luchsinger, J. A. (2009). Mediterranean diet and mild cognitive impairment. Archives of Neurology, 66(2), 216-225.

Schröder, H., Fitó, M., Estruch, R., Martínez-González, M. A., Corella, D., Salas-Salvadó, J., et al. (2011). A short screener is valid for assessing Mediterranean diet adherence among older Spanish men and women. The Journal of Nutrition, 141(6), 1140-1145.

Shin, J., \& Mattila, A. S. (2019). When organic food choices shape subsequent food choices: the interplay of gender and health consciousness. International Journal of Hospitality Management, 76, 94-101.

Sobal, J. (2005). Men, meat, and marriage: models of masculinity. Food and Foodways, 13(1-2), 135-158.

Spaan, P. E., Raaijmakers, J. G., \& Jonker, C. (2003). Alzheimer's disease versus normal ageing: a review of the efficiency of clinical and experimental memory measures. Journal of Clinical and Experimental Neuropsychology, 25(2), 216-233.
Sterne, J. A., Sutton, A. J., Ioannidis, J. P., Terrin, N., Jones, D. R., Lau, J., et al. (2011). Recommendations for examining and interpreting funnel plot asymmetry in meta-analyses of randomised controlled trials. Bmj, 343, d4002.

Tangney, C. C., Kwasny, M. J., Li, H., Wilson, R. S., Evans, D. A., \& Morris, M. C. (2011). Adherence to a Mediterranean-type dietary pattern and cognitive decline in a community population. The American Journal of Clinical Nutrition, 93(3), 601-607.

Tangney, C. C., Li, H., Wang, Y., Barnes, L., Schneider, J. A., Bennett, D. A., et al. (2014). Relation of DASH- and Mediterranean-like dietary patterns to cognitive decline in older persons. Neurology, 83(16), $1410-1416$.

Trichopoulou, A., Costacou, T., Bamia, C., \& Trichopoulos, D. (2003). Adherence to a Mediterranean diet and survival in a Greek population. The New England Journal of Medicine, 348(26), 2599-2608.

Trichopoulou, A., Kyrozis, A., Rossi, M., Katsoulis, M., Trichopoulos, D., La Vecchia, C., et al. (2014). Mediterranean diet and cognitive decline over time in an elderly Mediterranean population. European Journal of Nutrition, 54(8), 1311-1321. https://doi.org/10.1007/ s00394-014-0811-z.

Tsivgoulis, G., Judd, S., Letter, A. J., Alexandrov, A. V., Howard, G., Nahab, F., et al. (2013). Adherence to a Mediterranean diet and risk of incident cognitive impairment. Neurology, 80(18), 1684-1692.

Valls-Pedret, C., Sala-Vila, A., Serra-Mir, M., Corella, D., de la Torre, R., Martinez-Gonzalez, M. A., et al. (2015). Mediterranean diet and age-related cognitive decline: a randomized clinical trial. JAMA Internal Medicine, 175(7), 1094-1103.

Van Patten, R., Britton, K., \& Tremont, G. (2019). Comparing the MiniMental State Examination and the modified Mini-Mental State Examination in the detection of mild cognitive impairment in older adults. International Psychogeriatrics, 31(5), 693-701.

Vercambre, M. N., Grodstein, F., Berr, C., \& Kang, J. H. (2012). Mediterranean diet and cognitive decline in women with cardiovascular disease or risk factors. Journal of the Academy of Nutrition and Dietetics, 112(6), 816-823.

Wardle, J., Haase, A. M., Steptoe, A., Nillapun, M., Jonwutiwes, K., \& Bellisie, F. (2004). Gender differences in food choice: the contribution of health beliefs and dieting. Annals of Behavioral Medicine, 27(2), 107-116.

Willett, W. C., Sacks, F., Trichopoulou, A., Drescher, G., Ferro-Luzzi, A., Helsing, E., et al. (1995). Mediterranean diet pyramid: a cultural model for healthy eating. The American Journal of Clinical Nutrition, 61(6 Suppl), 1402s-1406s.

Wilson, R. S., Beckett, L. A., Bienias, J. L., Evans, D. A., \& Bennett, D. A. (2003). Terminal decline in cognitive function. Neurology, 60(11), 1782-1787.

Wood, W., \& Eagly, A. H. (2015). Two traditions of research on gender identity. Sex Roles, 73(11-12), 461-473.

Wu, L., \& Sun, D. (2017). Adherence to Mediterranean diet and risk of developing cognitive disorders: an updated systematic review and meta-analysis of prospective cohort studies. Scientific Reports, 7, 41317.

Yaffe, K. (2007). Metabolic syndrome and cognitive disorders: is the sum greater than its parts? Alzheimer Disease and Associated Disorders, 21(2), 167-171.

Publisher's Note Springer Nature remains neutral with regard to jurisdictional claims in published maps and institutional affiliations. 